\title{
Lesion Size Index in Maximum Voltage-Guided Cavotricuspid Ablation for Atrial Flutter
}

\author{
USAMA BOLES, MD ${ }^{1,2}$, ENES E. GUL, MD ${ }^{1}$, NOEL FITZPATRICK, MD², ANDRES ENRIQUEZ, MD ${ }^{1}$, \\ JOHN CONROY, MD ${ }^{2}$, ARIAN GHASSEMIAN, BHSC ${ }^{1}$, SANTHOSH DAVID, MD $^{3}$, \\ ADRIAN BARANCHUK, MD ${ }^{1}$, CHRISTOPHER SIMPSON, MD ${ }^{1}$, DAMIAN REDFEARN, MD ${ }^{1}$, \\ BENEDICT GLOVER, MD ${ }^{1}$, HOSHIAR ABDOLLAH, MD ${ }^{1}$ and KEVIN MICHAEL, MD ${ }^{1}$
}

\author{
${ }^{1}$ Division of Cardiology, Heart Rhythm Service, Queen's University, Kingston, Ontario, Canada \\ ${ }^{2}$ Department of Cardiology, Midland Regional Hospital and St. James Hospital, Trinity College, Dublin, Ireland \\ ${ }^{3}$ Department of Cardiology, Letterkenny University Hospital, Donegal, Ireland
}

\begin{abstract}
The application of optimum contact force (CF) can be used to improve ablation procedure success and safety. The lesion size index (LSI) is a novel dimensionless contact force parameter that allows for an accurate estimation of lesion volume in real time by integrating contact force (grams), duration (seconds) and power (watts). The aim was to correlate LSI values with current contact force parameters to achieve successful and safe bidirectional block of the cavotricuspid isthmus (CTI) using a maximum voltage-guided (MVG) ablation strategy. Fifteen consecutive patients (age $69 \pm 7.9$ years, nine males) with symptomatic atrial flutter (AFL) were evaluated and compared with 23 control (age $66.3 \pm 10.4$ years, 16 males) non-contact forceguided ablation cases. Irrigated-tip force-sensing ablation catheters (TactiCath Quart $z^{\mathrm{TM}}$, St. Jude Medical, St. Paul, MN, USA) were used in the CF group to achieve the primary endpoint of complete bidirectional block of the isthmus. In the CF group, a total of 233 radiofrequency (RF) applications were examined. A mean LSI of $6.4 \pm 1.0$ correlated with a force-time integral (FTI) of $581.2 \pm 230.9 \mathrm{~g} / \mathrm{s}$ and an average CF of $13.9 \pm 4.9 \mathrm{~g}$ concurrently. Intraprocedural, fluoroscopy time and RF time demonstrated lower trends in the CF group, but no significance with respect to these trends was observed. The secondary endpoint of no reconnection within 20 min after the procedure was equally attained in both groups, and, likewise, the level of safety was comparable. An LSI value of $>5$ represents a new effective parameter in MVG ablation for the cavotricuspid region that demonstrates a safe profile. Guidance of CTI ablation using LSI and other contact force parameters of CF $13.9 \pm 4.9 \mathrm{~g}$ and FTI $581.2 \pm 230.9 \mathrm{~g} / \mathrm{s}$ demonstrated highly effective and safe outcomes.
\end{abstract}

KEYWORDS. Ablation, atrial flutter, contact force, lesion size index, maximum voltage-guided.
ISSN 2156-3977 (print) ISSN 2156-3993 (online) CC BY 4.0 license

(C) 2017 Innovations in Cardiac Rhythm Management

\section{Introduction}

Radiofrequency (RF) ablation is a first-line therapy in atrial flutter and carries a superior success rate as compared with that of antiarrhythmic drug use. ${ }^{1}$ The use of optimum contact force $(\mathrm{CF})$ during catheter ablation is

The authors report no conflicts of interest for the published content. Manuscript received March 9, 2017. Final version accepted May 8, 2017.

Address correspondence to: Usama Boles, MD, 12 Colpe Park, Deepforde, Co. Meath, Ireland AA92ED0H.

E-mail: bolesu@tcd.ie. associated with increased transmural lesion volumes. CF sensing technology has provided additional ability to assess the level of applied force, enabling safe catheter manipulation and optimal clinical outcomes. ${ }^{2}$ Real-time assessment of $\mathrm{CF}$ is important given that higher $\mathrm{CF}$ may be potentially hazardous if the median forces exceed $60 \mathrm{~g}$ in patients with atrial fibrillation $(\mathrm{AF}){ }^{3}$

The contact force-time integral (FTI) has been employed as an endpoint to inform operators regarding successful lesion creation within the left atria. The FTI reflects the duration (in seconds) of the average contact force 
(in grams) applied during RF application, with lesion size correlating linearly with measured FTI (grams/ seconds). ${ }^{4}$ A cut-off of $400 \mathrm{~g} / \mathrm{s}$ or more for FTI has been associated with a higher success rate and lower recurrence of pulmonary vein reconnection compared with an FTI of lower than $400 \mathrm{~g} / \mathrm{s}$. Hence, an FTI $\geq 400 \mathrm{~g} / \mathrm{s}$ has been suggested as a surrogate end-point to predict the creation of transmural lesions in $\mathrm{RF}^{5,6}$

Booth et al. recently highlighted the new real-time CF parameter of lesion size index (LSI). This novel parameter incorporates RF power, ablation duration, and $\mathrm{CF}$ in a dimensionless ratio independent of traditional parameters. Clinically, LSI correlates with satisfactory short- to medium-term results. ${ }^{7}$ However, this novel CF marker has not yet been fully investigated in cavotricuspid isthmus (CTI) ablation.

We elected to investigate the use of LSI during maximum voltage-guided (MVG) ablation of typical atrial flutter (AFL), which is now accepted as a standard ablation strategy in many centers. It aims to concentrate the creation of ablation lesions on the muscle bundles responsible for transisthmus conduction. This method has the advantages of requiring significantly less RF ablation time and procedure time, as it does not necessitate the completion of the CTI line anatomically, as is done in the traditional "drag" technique. 8,9

Modern RF CTI ablation techniques for typical AFL have already been shown to be safe and effective. ${ }^{1}$ However, we speculate that the utilization of real-time operative parameters incorporating power, $\mathrm{CF}$ and time can still offer incremental benefits in terms of outcomes, safety and resource utilization. LSI is one such parameter; in this consecutive case series with controls, we consider the acute success rates, procedure time, fluoroscopy time and RF time. We further speculate that an LSI threshold of 5 may represent an appropriate intraoperative surrogate goal for the safe formation of transmural ablation lesions to achieve successful acute bidirectional block.

\section{Methods}

This was a retrospective single-center study that enrolled 15 consecutive patients who underwent CTI ablation for typical AFL using contact force-enabled catheters, and 23 patient controls who underwent ablation without the use of contact force-enabled catheters. All procedures were conducted under conscious sedation using midazolam and fentanyl boluses intravenously, and local anesthesia using lidocaine $1 \%$, and were performed by two expert operators who carry out a minimum of 100 ablation procedures per year. The study protocol was reviewed and approved by the institutional ethics committee of Kingston General Hospital and Queen's University in Ontario, Canada, and conformed to the ethical principles outlined by the Declaration of Helsinki.

\section{Patient demographics}

The controls for this study were randomly selected using age- and gender matching from patients admitted to
Kingston General Hospital for catheter ablation for typical AFL between January 2014 and December 2015. All control subjects met the selection criteria, and were enrolled in consecutive fashion.

We included patients $>18$ years old with documented paroxysmal or persistent AFL in this study, and excluded those who (1) had undergone a previous ablation procedure for AFL; (2) any non-CTI-dependent or atypical $\mathrm{AFL}$, (3) concomitant atrial fibrillation (AF); and/or (4) if they were unwilling to participate in the study.

Patient electronic charts were surveyed for demographics, comorbid conditions, history of cardiac disease, CHADS2 and CHAD2S2-VASc scores, and echocardiographic parameters prior to ablation, including left atrium diameter and left ventricular ejection fraction.

\section{Catheter set-up}

Standard intracardiac catheters were introduced through the patients' right femoral veins as appropriate for the procedure as follows: (1) first, Decapolar CSL ${ }^{\mathrm{TM}}$ catheters (St. Jude Medical, St. Paul, MN, USA) were presented. Next, (2) a 20-pole catheter was positioned along either the right atrial free wall or the tricuspid annulus, and (3) a mapping and ablation catheter (either TactiCath Quartz ${ }^{\mathrm{TM}}$, St. Jude Medical, St. Paul, MN, USA; or CoolFlex ${ }^{\mathrm{TM}}$, St. Jude Medical, St. Paul, MN, USA) was delivered either through a 9F femoral sheath (St. Jude Medical, Minneapolis, MN, USA) or (4) an Agilis ${ }^{\mathrm{TM}}$ steerable sheath (St. Jude Medical, Minneapolis, MN, USA), which was used in some cases for better stability or longer reach to the tricuspid valve annulus. (5) Finally, a quadripolar catheter (Supreme $^{\mathrm{TM}}$, St. Jude Medical, St. Paul, MN, USA) was placed at the right ventricular (RV) apex.

\section{Contact force and ablation procedures}

Prior to mapping, the contact force-enabled catheter (TactiCath Quartz ${ }^{\text {IM }}$, St. Jude Medical, St. Paul, MN, USA) was calibrated either outside the body or while floating freely in the mid-right atrium, to set the baseline value of contact force at zero grams. Mapping for maximum voltage electrograms (MVEs) was performed first, prior to $\mathrm{RF}$ ablation. A 4-mm CoolFlex ${ }^{\mathrm{TM}}$ irrigated catheter (St. Jude Medical, St. Paul, MN, USA) was used for ablation in the control group. Power delivery of RF was adjusted to $35 \mathrm{~W}$ with catheter irrigation set at $25 \mathrm{ml} / \mathrm{min}$, with $0.9 \%$ $\mathrm{NaCl}$.

The MVG RF was applied in both groups until complete bidirectional block was achieved. For each application of RF energy, the following data were extracted: application duration, average CF, FTI, LSI, and the number of RF lesions created. Information on the secondary endpoint of no reconnection after $20 \mathrm{~min}$ was also recorded, and any reconnections that occurred were noted.

Procedural duration, fluoroscopy duration, radiofrequency time, and the period of time after the last RF lesion were recorded. The MVE in millivolts $(\mathrm{mV})$ was identified as a target for initial RF application. AFL cycle 
Table 1: Demographic and Clinical Characteristics of the Patients

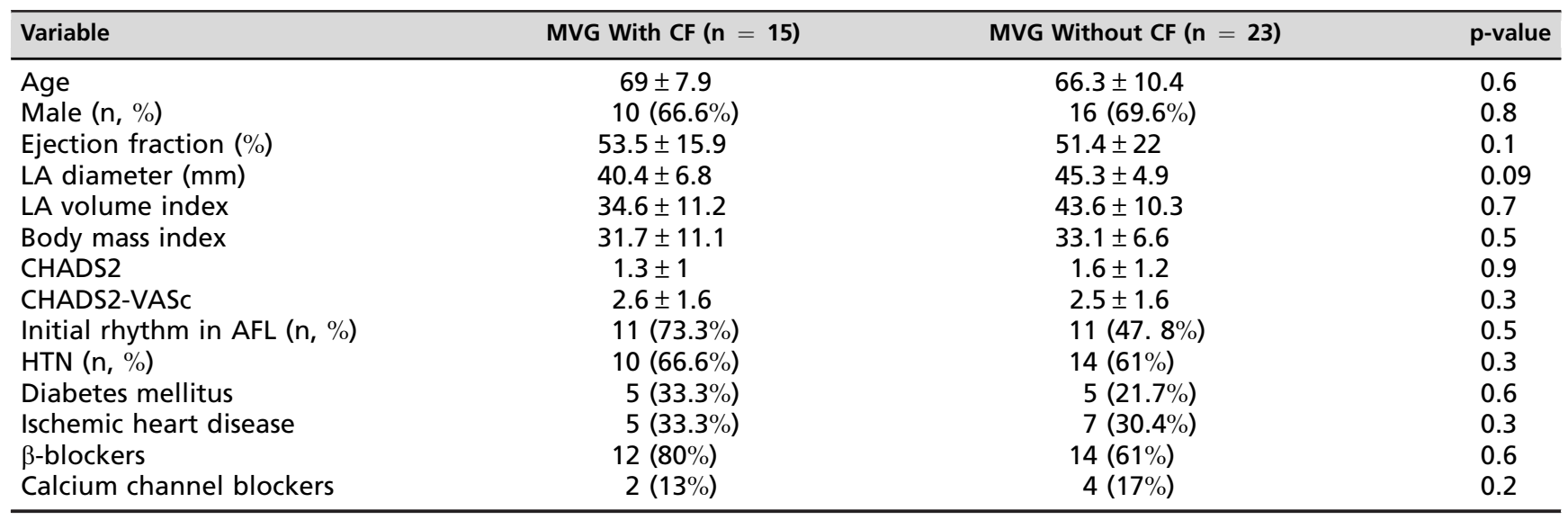

CF: contact force; n: number; AFL: atrial flutter; HTN: hypertension; LA: left atrium.

length (in milliseconds; $\mathrm{mm}$ ) was measured in those patients who were demonstrating AFL at the time of presentation to the clinic.

\section{Statistical analysis}

Data were analyzed using SPSS software version 21.0 (IBM Co., Armonk, NY, USA) and are presented as mean \pm standard deviation (SD) and median (interquartile range (IQR)) values. The distribution of the variables was analyzed with the Kolmogorov-Smirnov test. Differences between the two groups were tested using independent Student's t-tests for normally distributed variables, and the MannWhitney $U$ test for non-parametrically distributed variables. Differences between categorical variables were analyzed using the chi-squared test. A p-value of less than 0.05 was considered to be statistically significant.

\section{Results}

\section{Patient demographics}

A total of 15 patients were enrolled in this study as part of the CF group, versus 23 individuals in the non-CF group. All patients underwent MVE-guided RF ablation. Patient baseline characteristics are outlined in Table 1, with no differences between the study cohorts and controls noted.

\section{Contact force parameters and procedural details}

The LSI parameter for lesion depth and volume was $6.4 \pm 1.0$ (Figure 1). The overall average of CF was $13.9 \pm 4.9 \mathrm{~g}$ and the average of FTI was $581.2 \pm 230.9 \mathrm{~g} / \mathrm{s}$ (Table 2 and Figure 2). The results of both LSI and FTI are well correlated $(\mathrm{r}=0.8$ and $\mathrm{p}<0.001)$ (Figure 3 ). A trend of lower procedure time, fluoroscopy time, and RF time was observed in the CF group (Table 3). A successful secondary endpoint of no reconnection after 20 min was achieved in all patients, with no complications (Table 3).

\section{Intraoperative details}

Table 3 presents intraprocedural details for both groups. In the CF group, trends of lower RF time $(p=0.6)$, number of ablation lesions, fluoroscopy time, and, subsequently, procedure time, were noted.

\section{AFL at presentation}

Eleven patients in the CF group and 12 patients in the non-CF group had AFL as initial rhythm at the beginning of their respective procedures. AFL cycle length, the time to terminate AFL, the number of lesions required, and MVE are outlined in Table 4. It is notable that there was a trend in the CF group that led to a lower number of required RF lesions and, subsequently, a shorter time to termination of AFL.

\section{Discussion}

Although AFL ablation is already a highly successful and safe procedure associated with low recurrence rates, ${ }^{1}$ a number of developed technological and methodological innovations have offered possible incremental benefits in terms of efficiency without sacrificing safety or effectiveness.

Redfearn et al. described an ablation strategy that targets MVE in CTI ablation (ie, critical conducting bundles) that has demonstrated similar success rates to the traditional anatomical CTI line formation (the so-called "drag" technique), but which necessitates fewer ablation lesions and lower radiation exposure times. ${ }^{8,9}$ In our cohorts, the number of lesions required to achieve bidirectional block was $8.3 \pm 7.6$ in the CF group and $13.2 \pm 9$ in the control (non-CF) group.

The EFFICAS I and II studied identified optimal contact force parameters resulting in more durable pulmonary vein isolation (PVI) in catheter ablation of paroxysmal AF (PAF). These parameters were associated with a lower incidence of gap formation (ie, they achieved effective lesion formation) and, hence, a lower recurrence rate. The recommendations of CF parameters in PVI procedures include a target average CF value of $10-30 \mathrm{~g}$ and a 


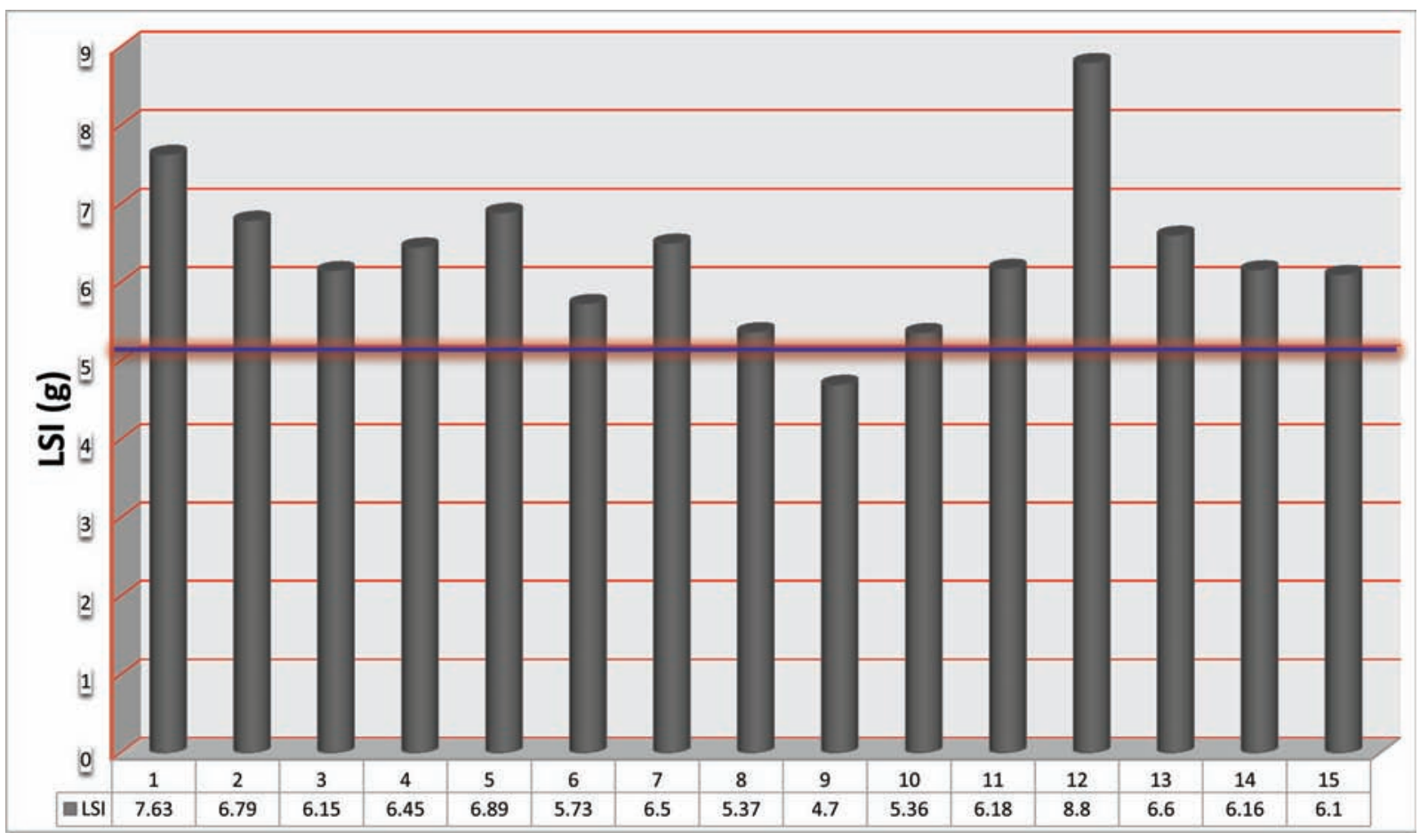

Figure 1: The mean LSI reached in each procedure. A horizontal line here illustrates that the minimum average of LSI $\geq 5 \mathrm{~g}$ was predominantly reached.

Table 2: CF Parameters Achieved During the Procedure

\begin{tabular}{lccc}
\hline Variable & Mean \pm SD & Minimum & Maximum \\
\hline CF average $(\mathrm{g})$ & $13.9 \pm 4.9$ & 7.9 & 22.2 \\
FTI $(\mathrm{g} / \mathrm{s})$ & $581.2 \pm 230.9$ & 324.9 & 1300.4 \\
LSI & $6.4 \pm 1.0$ & 1.47 & 8.8 \\
\hline
\end{tabular}

SD: standard deviation; CF: contact force; FTI: force-time integral; LSI: lesion size index.

minimum FTI value of $400 \mathrm{~g}$ for each RF lesion. . $^{5,14,15}$ Another landmark study, TOCCASTAR by Reddy et al., confirmed the importance of using CF-guided strategies in meeting primary safety and effectiveness endpoints in PVI ablation procedures. ${ }^{16}$ In results published by Neuzil et al., ${ }^{10}$ evidence has been offered that supports the concept that an LSI of greater than 5 predicts reconnection risk in PVI ablation. Although the above studies relate to PVI/PAF ablation, it is reasonable to use these results as a guide at least in qualitative, if not quantitative terms, when considering CTI/AFL ablation.

Successful RF lesion formation has been shown to be dependent on ablation lesion size and volume, which is associated with good tissue catheter contact and time of RF application. ${ }^{3,17,18}$ Intuitively, successful lesion formation is related to the amount of RF energy transmitted to the prospective lesion site, and is a function of contact force, contact surface area, application time, and application power. Real-time operative parameters to date have considered CF and application time (combined in FTI); however, LSI also incorporates power along with CF and time in a nonlinear fashion to provide a novel dimensionless parameter to guide ablation techniques. Both LSI and FTI are complimentary parameters, yet LSI incorporates the power delivered during RF application (Figure 4).

In this study, we consider the possible impact of using LSI alongside other previously validated real-time operative parameters (CF and FTI) during MVG CTI ablation for typical AFL. In particular, when aiming for an LSI $>5$, we show that an LSI of $6.4 \pm 1.0$ reflected a surrogate marker for successful transmural lesion formation, leading to acute bidirectional block of CTI. Although there was no statistically significant difference seen in the intraprocedural data between our study group and the controls, there were trends of less RF application time, fewer ablation lesions, and shorter fluoroscopy and overall procedure times in the LSI/CF-guided patients. Thus, LSI may be reflected as a sufficient parameter once the value of 5 is reached. This LSI value ensures sufficient transmural RF penetration with a safe outcome. ${ }^{9-13}$

\section{Study limitations}

This was a single-center study that involved a small number of patients; therefore, larger randomized studies with power analysis are needed to validate these findings. Additionally, our learning curve with CF catheter use may also influence the results. Furthermore, the aim of this study was to evaluate the effects of LSI and CF parameter use in order to achieve acute procedural success only, and, thus, no follow-up data were included. Future studies incorporating medium- to long-term 


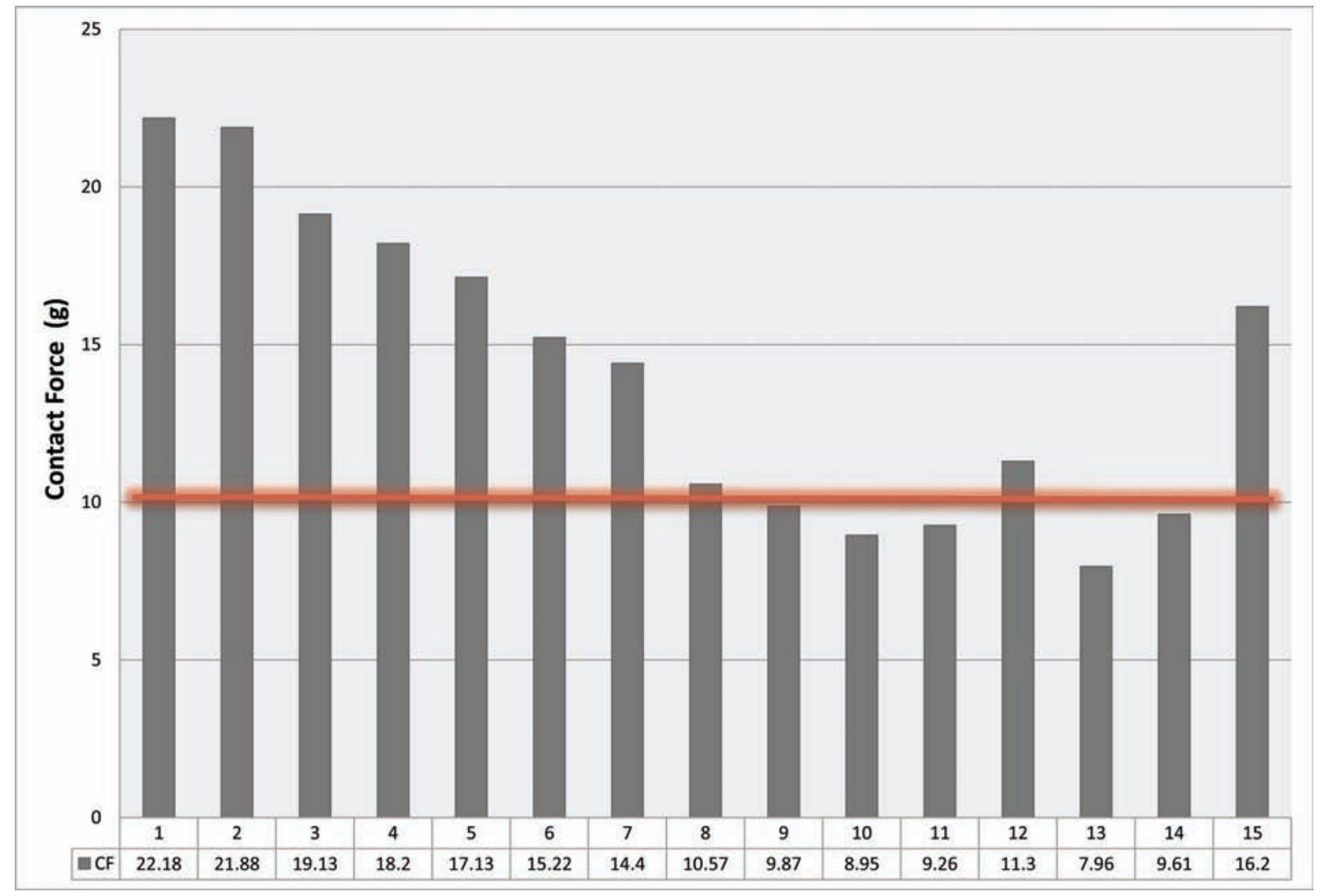

Figure 2: The mean of average CF values reached. A horizontal line illustrates the minimum average $\mathrm{CF} \geq 10 \mathrm{~g}$, which was predominantly reached.

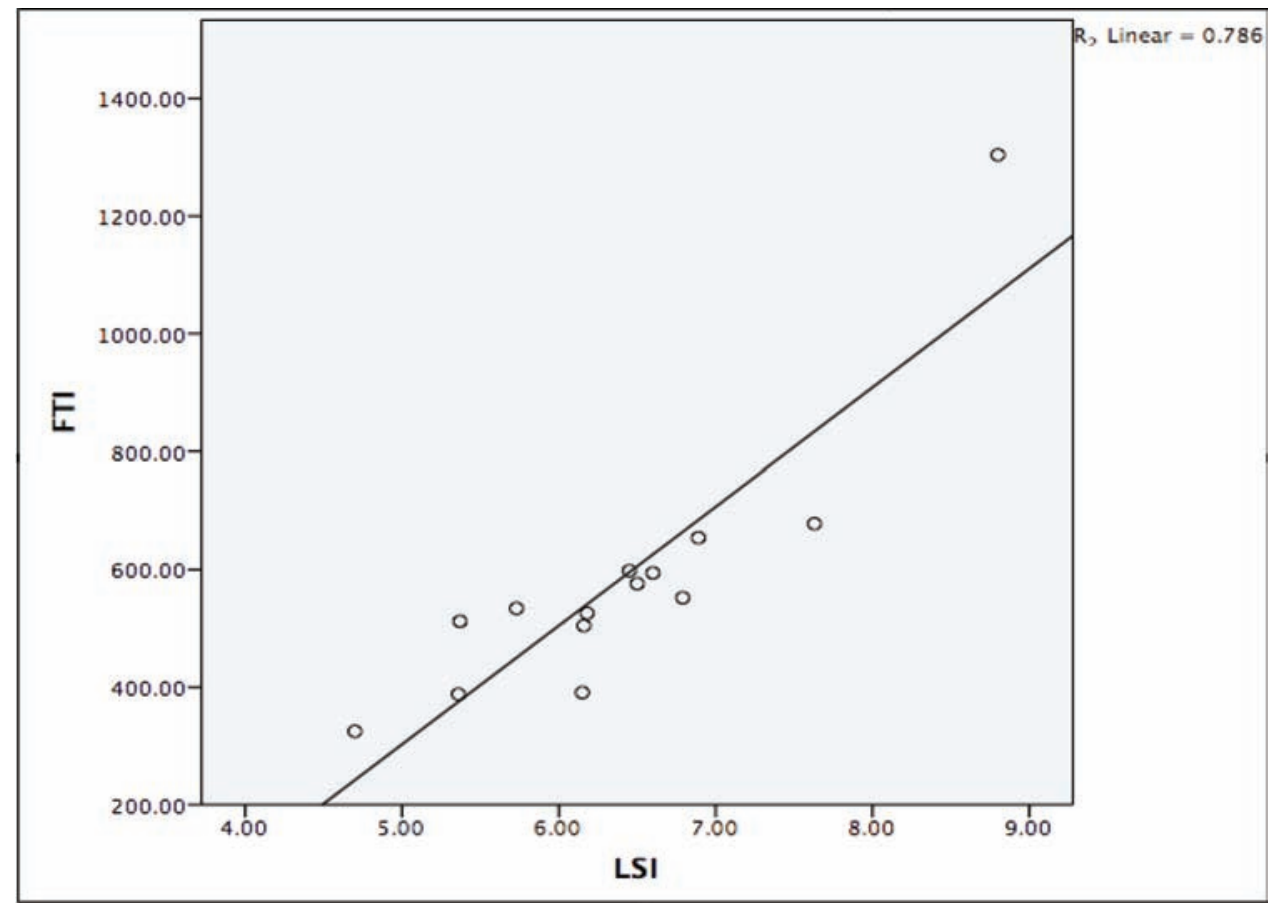

Figure 3: This figure depicts the correlations between LSI and FTI. This correlation was examined in contact force group using TactiCath $^{\mathrm{TM}}$ (St. Jude Medical, St. Paul, MN, USA) catheters. 
Table 3: Intraprocedural Data Between the Two Groups

\begin{tabular}{|c|c|c|c|}
\hline Variable & MVG TactiCath ${ }^{\mathrm{TM}}$ CF $(n=15)$ & MVG (Non-CF) $(n=23)$ & p-value \\
\hline RF time (min) & $5.1 \pm 2.3$ & $7.6 \pm 4.8$ & 0.06 \\
\hline Ablation lesions ( $n$ ) & $8.3 \pm 7.6$ & $13.2 \pm 9$ & 0.4 \\
\hline Fluoroscopy time (min) & $10.8 \pm 8.9$ & $12.3 \pm 12.1$ & 0.36 \\
\hline Procedure time (min) & $87.7 \pm 24.2$ & $93.2 \pm 46.3$ & 0.35 \\
\hline MVE (mV) & $1.86 \pm 1.4$ & $1.55 \pm 1.4$ & 0.3 \\
\hline Reconnection after $20 \min (n, \%)$ & $2(13 \%)$ & $2(8.6 \%)$ & 0.5 \\
\hline
\end{tabular}

CF: contact force; n: number; RF: radiofrequency; MVE: maximum voltage electrogram.

Table 4: Subanalysis of the Group of Patients Who Presented with AFL at the Start of the Procedure

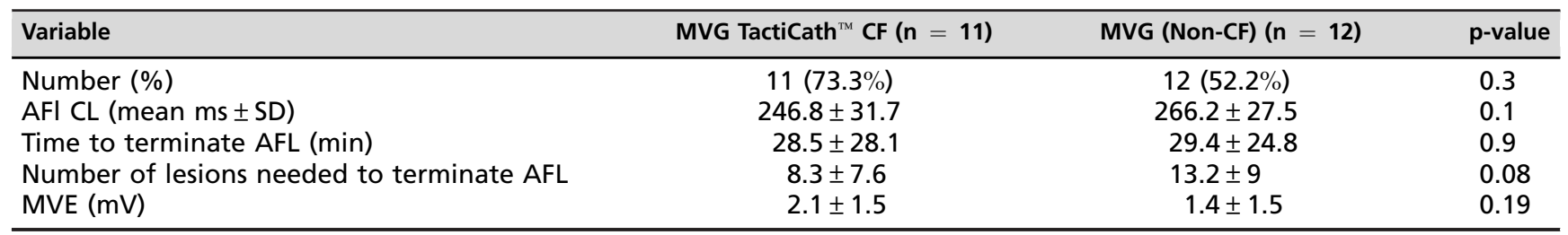

CF: contact force; n: number; AFL: atrial flutter; CL: cycle length; MVE: maximum voltage electrogram.

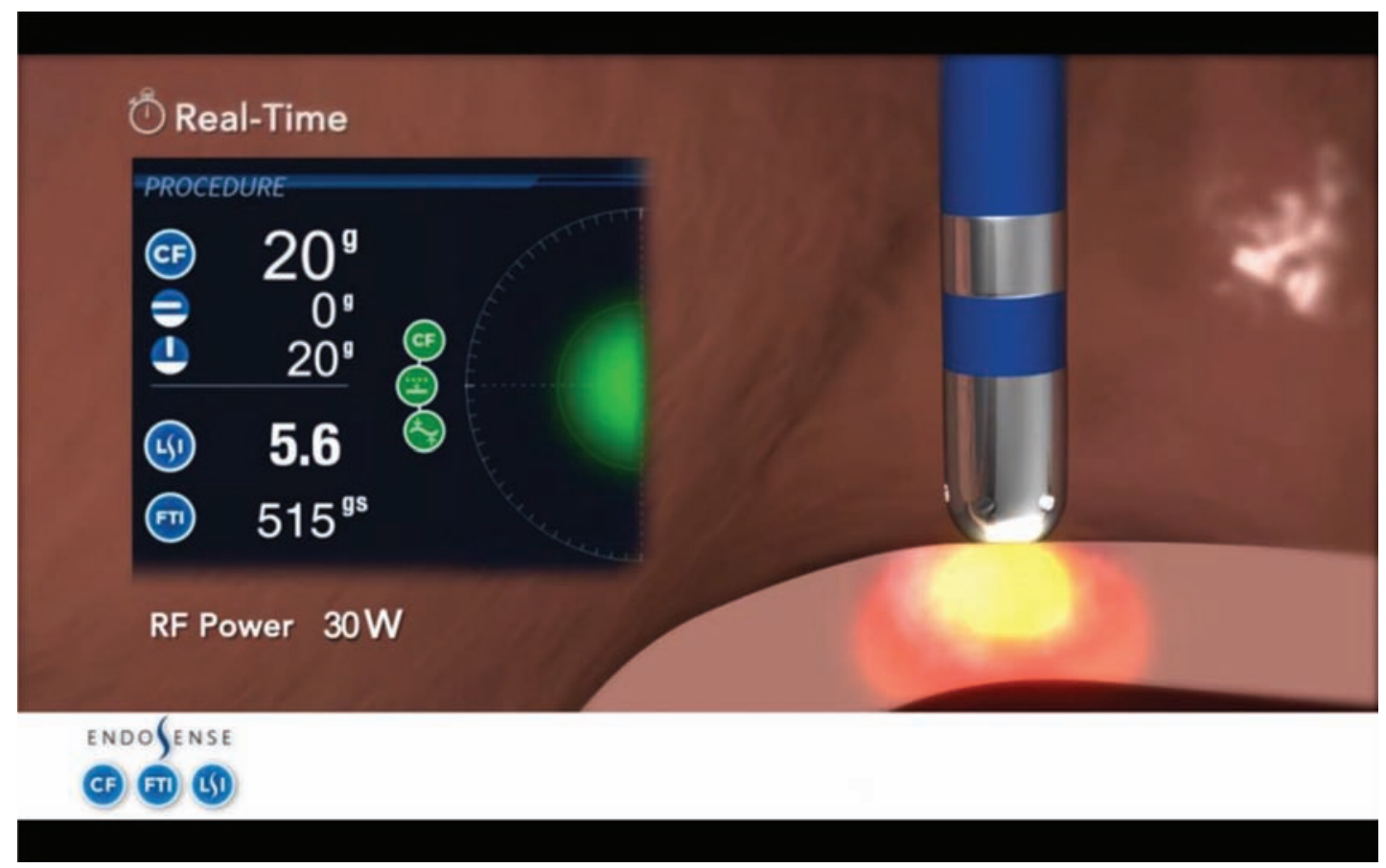

Figure 4: A force-sensing ablation catheter (TactiCath ${ }^{\mathrm{TM}}$, St. Jude Medical, St. Paul, MN, USA) showing real-time CF parameters, including CF, LSI and FTI during RF application. Courtesy of Endosense.

follow-up with patients could prove to be a valuable resource.

\section{Conclusions}

In MVG cavotricuspid ablation, an LSI $>5(6.4 \pm 1.0)$ generated an average CF value of $13.9 \pm 4.9 \mathrm{~g}$ and an FTI of $581.2 \pm 230.9 \mathrm{~g} / \mathrm{s}$. The use of these parameters led to an acute success rate and safe application similar to that of the standard accepted techniques. Current experience using LSI is early, and more studies are required to validate it.

\section{References}

1. Natale A, Newby KH, Pisano E, et al. Prospective randomized comparison of antiarrhythmic therapy versus firstline radiofrequency ablation in patients with atrial flutter. J Am Coll Cardiol. 2000;35(7):1898-1904. 
2. Kuck KH, Reddy VY, Schmidt B, et al. A novel radiofrequency ablation catheter using contact force sensing: Toccata study. Heart Rhythm. 2012;9(1):18-23.

3. Ikeda A, Nakagawa $H$, Lambert $H$, et al. Relationship between catheter contact force and radiofrequency lesion size and incidence of steam pop in the beating canine heart: electrogram amplitude, impedance, and electrode temperature are poor predictors of electrode-tissue contact force and lesion size. Circ Arrhythm Electrophysiol. 2014;7(6): 1174-1180.

4. Shah DC, Lambert $H$, Nakagawa $H$, Langenkamp A, Aeby N, Leo G. Area under the real-time contact force curve (force-time integral) predicts radiofrequency lesion size in an in vitro contractile model. J Cardiovasc Electrophysiol. 2010;21(9):1038-1043.

5. Kautzner J, Neuzil P, Lambert H, et al. EFFICAS II: optimization of catheter contact force improves outcome of pulmonary vein isolation for paroxysmal atrial fibrillation. Europace. 2015;17(8):1229-1235.

6. Squara F, Latcu DG, Massaad Y, Mahjoub M, Bun SS, Saoudi $\mathrm{N}$. Contact force and force-time integral in atrial radiofrequency ablation predict transmurality of lesions. Europace. 2014;16(5):660-667.

7. Booth C, Ng K, Dauber K, Kaye G, Gould P. The guidance of cavo-tricuspid isthmus ablation using a contact force ablation catheter and novel parameters [Abstract 328]. Europace. 2015; 17(S 3):40.

8. Redfearn DP, Skanes AC, Gula LJ, Krahn AD, Yee R, Klein GJ. Cavotricuspid isthmus conduction is dependent on underlying anatomic bundle architecture: observations using a maximum voltage-guided ablation technique. J Cardiovasc Electrophysiol. 2006;17(8):832-838.

9. Gula LJ, Redfearn DP, Veenhuyzen GD, et al. Reduction in atrial flutter ablation time by targeting maximum voltage: results of a prospective randomized clinical trial. J Cardiovasc Electrophysiol. 2009;20(10):1108-1112.

10. Neuzil P KK, Nakagawa H, Kautzner J, Shah DC, Fremont O. Lesion size index for prediction of reconnection risk following RF ablation for PVI [Abstract MP04-03]. Heart Rhythm. 2012;9(5):S492.

11. Gould PA, Booth C, Dauber K, Ng K, Claughton A, Kaye GC. Characteristics of cavotricuspid isthmus ablation for atrial flutter guided by novel parameters using a contact force catheter. J Cardiovasc Electrophysiol. 2016;27(12): $1429-1439$.
12. Venier S, Andrade JG, Khairy P, et al. Contact-force-guided vs. contact-force-blinded catheter ablation of typical atrial flutter: a prospective study. Europace. 2016 Jul 4. pii: euw137. [Epub ahead of print].

13. Rodenberg $\mathrm{C}$, Zhou XH. ROC curve estimation when covariates affect the verification process. Biometrics. 2000; 56(4):1256-1262.

14. Neuzil P, Reddy VY, Kautzner J, et al. Electrical reconnection after pulmonary vein isolation is contingent on contact force during initial treatment: results from the EFFICAS I study. Circ Arrhythm Electrophysiol. 2013;6(2):327-333.

15. Natale A, Reddy VY, Monir G, et al. Paroxysmal AF catheter ablation with a contact force sensing catheter: results of the prospective, multicenter SMART-AF trial. J Am Coll Cardiol. 2014;64(7):647-656.

16. Reddy VY, Dukkipati SR, Neuzil P, et al. Randomized, controlled trial of the safety and effectiveness of a contact force-sensing irrigated catheter for ablation of paroxysmal atrial fibrillation: results of the TactiCath Contact Force Ablation Catheter Study for Atrial Fibrillation (TOCCASTAR) study. Circulation. 2015;132(10):907-915.

17. Kalman JM, Fitzpatrick AP, Olgin JE, et al. Biophysical characteristics of radiofrequency lesion formation in vivo: dynamics of catheter tip-tissue contact evaluated by intracardiac echocardiography. Am Heart J. 1997;133(1):8-18.

18. Bosnos M, Guillen-Rodriguez JM, He DS, Marcus FI. Early assessment of biophysical parameters predicts lesion formation during RF energy delivery in vitro. Pacing Clin Electrophysiol. 2010;33(9):1082-1088.

19. Mansour M SD, Kalbfleisch S. Application of contact force guidelines increases durable isolation after pulmonary vein isolation for paroxysmal atrial fibrillation. Heart Rhythm J. 2013;8(S):133.

20. Kautzner J, Natale G, Michaud G, et al. Segmental variability in lesion size is controlled using contact force during pulmonary venous isolation [Abstract P523]. Europace. 2013; 15(Suppl 2):S56.

21. Haldar S, Jarman JW, Panikker S, et al. Contact force sensing technology identifies sites of inadequate contact and reduces acute pulmonary vein reconnection: a prospective case control study. Int J Cardiol. 2013;168(2):1160-1166.

22. Sacher F, Wright M, Derval N, et al. Endocardial versus epicardial ventricular radiofrequency ablation: utility of in vivo contact force assessment. Circ Arrhythm Electrophysiol. 2013;6(1):144-145. 